\title{
Reading Mindset Measure
}

Stephanie Al Otaiba, Brenna Rivas, and Francesca Jones

Southern Methodist University

Yaacov Petscher

Florida State University

Jeanne Wanzek

Vanderbilt University

(2015) Unpublished test, College of Education, Southern Methodist University, Dallas, TX.

\begin{abstract}
Author Notes
We have no known conflict of interest to disclose. The research reported here was supported by the Institute of Education Sciences, U.S. Department of Education, through Grant R324A130262 to Vanderbilt University. The opinions expressed are those of the authors and do not represent views of the Institute or the U.S. Department of Education.

Correspondence concerning this article should be addressed to Stephanie Al Otaiba, Professor, Department of Teaching and Learning, Southern Methodist University, P.O. Box 750445, Dallas, TX 75275-0455. Email:salotaiba@smu.edu Stephanie Al Otaiba https://orcid.org/0000-0001-
\end{abstract} $7125-3791$ 


\begin{tabular}{lcccccc} 
& $\begin{array}{c}\text { DISAGREE } \\
\text { A LOT }\end{array}$ & DISAGREE & $\begin{array}{c}\text { DISAGREE } \\
\text { A LITTLE }\end{array}$ & $\begin{array}{c}\text { AGREE } \\
\text { A LITTLE }\end{array}$ & AGREE & $\begin{array}{c}\text { AGREE } \\
\text { A LOT }\end{array}$ \\
\hline If a book is hard to read, I stop reading it. & $\square$ & $\square$ & $\square$ & $\square$ & $\square$ & $\square$ \\
\hline $\begin{array}{l}\text { I feel like I am one of the worst readers in } \\
\text { my class. }\end{array}$ & $\square$ & $\square$ & $\square$ & $\square$ & $\square$ & $\square$ \\
\hline $\begin{array}{l}\text { If I have to read out loud in class, I feel } \\
\text { scared. }\end{array}$ & $\square$ & $\square$ & $\square$ & $\square$ & $\square$ & $\square$ \\
\hline $\begin{array}{l}\text { If I make a lot of mistakes while reading, I } \\
\text { quit trying. }\end{array}$ & $\square$ & $\square$ & $\square$ & $\square$ & $\square$ & $\square$ \\
\hline $\begin{array}{l}\text { When I have to work ard at reading, it } \\
\text { makes me feel like I am not very smart. }\end{array}$ & $\square$ & $\square$ & $\square$ & $\square$ & $\square$ & $\square$ \\
\hline $\begin{array}{l}\text { When someone reads better than me, I'm } \\
\text { jealous. }\end{array}$ & $\square$ & $\square$ & $\square$ & $\square$ & $\square$ & $\square$ \\
\hline $\begin{array}{l}\text { I don't like when my teacher corrects me } \\
\text { when I'm reading. }\end{array}$ & $\square$ & $\square$ & $\square$ & $\square$ & $\square$ & $\square$ \\
\hline
\end{tabular}




\section{References}

Tock, J., Quinn, J., Al Otaiba, S., Petscher, Y., Wanzek, J. (in press). Establishing a Reading Mindset Measure: A Validation Study. Assessment for Effective Instruction.

Petscher, Y., Al Otaiba, S., Wanzek, J., Rivas. B., \& Jones, F. (2017). The relation between global and specific mindset with reading outcomes for elementary school students. Scientific Studies of Reading, 21:5, 376-391, doi: 10.1080/10888438.2017.1313846 